Article

\title{
A Molecular Genetic Linkage Map of Eucommia ulmoides and Quantitative Trait Loci (QTL) Analysis for Growth Traits
}

\author{
Yu Li ${ }^{1}$, Dawei Wang ${ }^{1,2}$, Zhouqi Li ${ }^{1, *}$, Junkun Wei ${ }^{1}$, Cangfu Jin ${ }^{1}$ and Minhao Liu ${ }^{1}$ \\ 1 College of Forestry, Northwest A\&F University, Yangling 712100, Shaanxi, China; \\ E-Mails: liyu@nwsuaf.edu.cn (Y.L.); daweiwon@163.com (D.W.); jun_kun@126.com (J.W.); \\ jincangfu2008@nwsuaf.edu.cn (C.J.); liuminhao@nwsuaf.edu.cn (M.L.) \\ 2 College of Forestry, Southwest Forestry University, Kunming 650224, Yunnan, China \\ * Author to whom correspondence should be addressed; E-Mail: lizhouqi@nwsuaf.edu.cn; \\ Tel./Fax: +86-29-8708-2992.
}

Received: 6 December 2013; in revised form: 19 January 2014 / Accepted: 21 January 2014 / Published: 28 January 2014

\begin{abstract}
Eucommia ulmoides is an economically important tree species for both herbal medicine and organic chemical industry. Effort to breed varieties with improved yield and quality is limited by the lack of knowledge on the genetic basis of the traits. A genetic linkage map of E. ulmoides was constructed from a full-sib family using sequence-related amplified polymorphism, amplified fragment length polymorphism, inter-simple sequence repeat and simple sequence repeat markers. In total, 706 markers were mapped in 25 linkage groups covering $2133 \mathrm{cM}$. The genetic linkage map covered approximately $89 \%$ of the estimated E. ulmoides genome with an average of $3.1 \mathrm{cM}$ between adjacent markers. The present genetic linkage map was used to identify quantitative trait loci (QTL) affecting growth-related traits. Eighteen QTLs were found to explain $12.4 \%-33.3 \%$ of the phenotypic variance. This genetic linkage map provides a tool for marker-assisted selection and for studies of genome in E. ulmoides.
\end{abstract}

Keywords: Eucommia ulmoides; molecular markers; genetic linkage map; growth traits; QTL 


\section{Introduction}

Eucommia ulmoides Oliver $(2 n=34)$, the single extant species of the genus Eucommia (Eucommiaceae), is strictly a dioecious perennial tree [1]. It is an economically important plant for both herbal medicine and organic chemical industry. Chemical constituents (e.g., phenylpropanoids and flavonoids) in the bark and leaves have high pharmacological activities and health care functions of lowering blood pressure and blood sugar, resisting oxidation and mutation, improving the health, strengthening the body, promoting metabolism and relieving tiredness [2-5]. The whole plant except xylem contains Eucommia-rubber which is an important raw material in the chemical industry. Eucommia-rubber is a hard rubber with thermoplasticity, and it has properties that are similar to those of plastic [6]. Historically, only the bark was officially recognized as a traditional Chinese herbal drug. In recent years, the bark of E. ulmoides also was used to produce Eucommia-rubber in China, Russia and Japan. To improve the quality and yield of the bark, height and diameter growth were the main parameters for selection [7].

Conventional breeding of E. ulmoides has mainly focused on the selection of promising plants from existing natural populations. These selected plants were propagated vegetatively and released as clones. Recently, these cultivars were used as parents in crossbreeding. However, classical breeding often takes decades to fully evaluate and release new cultivars. The ability of $E$. ulmoides breeders to select promising parents for crossing, and to identify progenies with favorable combinations of characters, is hampered by the limited knowledge of the genetic basis of economically important traits. The speed and precision of breeding can be improved by the development of genetic linkage maps. Such genetic linkage maps can facilitate the development of diagnostic markers for polygenic traits and the identification of genes controlling complex phenotypes. The linked molecular markers identified in quantitative trait loci (QTL) analysis could then potentially be used in breeding practice via marker-assisted selection, where the selection is based on DNA sequence rather than the phenotype.

For forest trees, given the high genetic load and long generation time, segregating populations derived from crosses between inbred lines are not available. To circumvent this limitation, a pseudo-testcross approach is generally used to construct linkage maps from full-sib populations. Combined with the pseudo-testcross strategy, molecular markers such as random amplified polymorphic DNA (RAPD), sequence related amplified polymorphism (SRAP), amplified fragment length polymorphism (AFLP), inter-simple sequence repeat (ISSR) and simple sequence repeat (SSR) have been used extensively for the preparation of linkage maps of a number of tree species [8-12]. In a pseudo-testcross, only dominant markers that segregate in a 1:1 ratio are used to build separate molecular maps for each parent. Considering modern marker technologies are available for full-sib populations, markers that segregate in $3: 1$ (dominant), 1:2:1 (co-dominant) and 1:1:1:1 (co-dominant) ratios, in addition to $1: 1$, can be used to integrate individual linkage maps [13-15]. Using genetic linkage maps, QTL analysis have been conducted for traits of leaf, growth, vegetative propagation, wood quality, resistance, yield, flowering and fruiting in tree species [16-21].

In order to construct a genetic linkage map of E. ulmoides, we produced a $\mathrm{F}_{1}$ mapping population from the cross between a wild genotype Xiaoye and a cultivar Qinzhong No.1. The female parent Xiaoye originated from the forest at Yantuo, Lingbao, Henan. The male parent Qinzhong No.1 was one of the four earliest cultivars [22], and it was planted in the museum garden of Northwest A\&F 
University, Yangling, Shaanxi. Xiaoye and Qinzhong No.1 were chosen as parents because they differ in important quantitative traits. For instance, Xiaoye has late budding and flowering times, low content of secondary metabolite, small leaves, and smooth bark, whereas Qinzhong No.1 has early budding and flowering times, high content of secondary metabolite, large leaves, and rough bark. Besides, Qinzhong No.1 is an excellent cultivar, fast growing and with high resistance to drought and cold. In this study, we present a genetic linkage map of E. ulmoides based on SRAP, AFLP, ISSR and SSR markers. Results from our QTL analysis for height and basal diameter, measured over four consecutive years, are reported.

\section{Results}

\subsection{Molecular Markers}

The 2048 SRAP primer combinations (Table 1), 64 AFLP primer combinations (Table 2), 100 ISSR primers and 19 SSR primer combinations were tested on the parents and a small set of the DZ0901 progeny. Of these, 131 SRAP primer combinations, 18 AFLP primer combinations, 16 ISSR primers (Table 3) and 17 SSR primer combinations were more informative and were used for amplification (Table 4). Of the 1604 polymorphic SRAP markers (with an average of 12.2 polymorphic markers per primer combination), 305 were lmxll markers (1:1), 326 were nnxnp markers (1:1), 382 were hkxhk markers (3:1), 18 were hkxhk markers (1:2:1), 13 were egxef markers (1:1:1:1), eight were abxcd markers $(1: 1: 1: 1)$, and $552(34.4 \%)$ showed segregation distortion $(p<0.05)$ (Table 4$)$. Of the 295 polymorphic AFLP markers (with an average of 16.4 polymorphic markers per primer combination), 141 were lmxll markers (1:1), 108 were nnxnp markers (1:1), 22 were hkxhk markers (3:1), and 24 (8.1\%) showed segregation distortion $(p<0.05)$ (Table 4$)$. Of the 111 polymorphic ISSR markers (with an average of 6.9 polymorphic markers per primer), 27 were lmxll markers (1:1), 23 were nnxnp markers (1:1), 31 were hkxhk markers (3:1), and 30 (27.0\%) showed segregation distortion $(p<0.05)$ (Table 4). Of the 132 polymorphic SSR markers (with an average of 7.8 polymorphic markers per primer combination), 33 were lmxll markers (1:1), 42 were nnxnp markers (1:1), 26 were hkxhk markers $(3: 1)$, six were hkxhk markers $(1: 2: 1)$, seven were egxef markers $(1: 1: 1: 1)$, one were abxcd markers $(1: 1: 1: 1)$, and $17(12.9 \%)$ showed segregation distortion $(p<0.05)$ (Table 4$)$. In total, 2142 polymorphic markers were scored from 182 primer combinations or primers with an average of 11.8 polymorphic markers per primer combination or primers. Of these, 623 (29.0\%) markers showed segregation distortion $(p<0.05)$ and were excluded from mapping. Only 1519 markers conforming to Mendelian segregation ratios were used for the construction of the genetic linkage map.

\subsection{Genetic Linkage Map}

The genetic linkage map (DZ0901) consisted of 706 markers distributed over 25 linkage groups (LG) covering $2133 \mathrm{cM}$ (Table 5 and Figure 1). The number of mapped makers per LG ranged from 5-106 with a mean of 28.2. The map size of the LGs ranged from 19.9-194.0 cM with a mean of $85.3 \mathrm{cM}$. The average map distance between adjacent markers was $3.1 \mathrm{cM}$. In addition, 165 markers distributed over 25 triplets and 45 doublets. There were 628 unlinked markers and 20 markers that successfully linked with a group but could not be ordered. Since our estimate of 
E. ulmoides genome length was $2403 \mathrm{cM}$, the genetic linkage map constructed in our study covered approximately $89 \%$ of the genome.

Table 1. Primer sequences used in the sequence-related amplified polymorphism analysis.

\begin{tabular}{|c|c|c|c|c|c|}
\hline \multicolumn{4}{|c|}{ Forward primer } & \multicolumn{2}{|c|}{ Reverse primer } \\
\hline Name & Sequence & Name & Sequence & Name & Sequence \\
\hline mel & ATA & me33 & GAA & em1 & AAT \\
\hline me2 & $\mathrm{AGC}$ & me34 & GAT & em2 & TGC \\
\hline me3 & AAT & me35 & GAG & em 3 & GAC \\
\hline me4 & $\mathrm{ACC}$ & me36 & GAC & em4 & TGA \\
\hline me5 & AAG & me37 & GTA & em5 & $\mathrm{AAC}$ \\
\hline me6 & $\mathrm{ACA}$ & me38 & GTT & em6 & GCA \\
\hline me7 & ACG & me39 & GTG & em7 & CAA \\
\hline me8 & $\mathrm{ACT}$ & me 40 & GTC & em8 & $\mathrm{CAC}$ \\
\hline me9 & AGG & me41 & GGA & em9 & CAG \\
\hline me10 & $\mathrm{AAA}$ & me42 & GGT & em10 & CAT \\
\hline me11 & $\mathrm{AAC}$ & me43 & GGG & em11 & CTA \\
\hline me12 & AGA & me44 & GGC & em12 & CTC \\
\hline me13 & ATG & me45 & GCA & em13 & CTG \\
\hline me14 & ATC & me46 & GCT & em14 & CTT \\
\hline me15 & ATT & me47 & GCG & em15 & GAT \\
\hline me16 & AGT & me48 & GCC & em 16 & GTC \\
\hline me17 & TAA & me49 & CAA & em17 & AAG \\
\hline me18 & TAT & me50 & CAT & em18 & ATC \\
\hline me19 & TAG & me51 & CAG & em19 & AGA \\
\hline me20 & TAC & me52 & CAC & em20 & $\mathrm{ACT}$ \\
\hline me21 & TTA & me53 & CTA & em21 & TAC \\
\hline me22 & TTT & me54 & CTT & em22 & TTG \\
\hline me23 & TTG & me55 & CTG & em 23 & TGT \\
\hline me24 & TTC & me56 & CTC & em 24 & TCG \\
\hline me25 & TGA & me57 & CGA & em 25 & GAA \\
\hline me26 & TGT & me58 & CGT & em 26 & GTG \\
\hline me27 & TGG & me59 & CGG & $\mathrm{em} 27$ & GGA \\
\hline me28 & TGC & me60 & CGC & $\mathrm{em} 28$ & GCT \\
\hline me29 & TCA & me61 & CCA & em29 & CGA \\
\hline me30 & TCT & me62 & $\mathrm{CCT}$ & em30 & CGT \\
\hline me31 & TCG & me63 & CCG & em31 & $\mathrm{CCA}$ \\
\hline me32 & TCC & me64 & $\mathrm{CCC}$ & em32 & $\mathrm{CCT}$ \\
\hline
\end{tabular}

The primers consist of the core sequences and three selective nucleotides at the $3^{\prime}$ end. The core sequence of the forward primers is TGAGTCCAAACCGG. The core sequence of the reverse primers is GACTGCGTACGAATT. Only the three selective nucleotides are presented. A total of 2048 SRAP primer combinations were used to screen for polymorphisms. 
Table 2. Primer sequences used in the amplified fragment length polymorphism analysis.

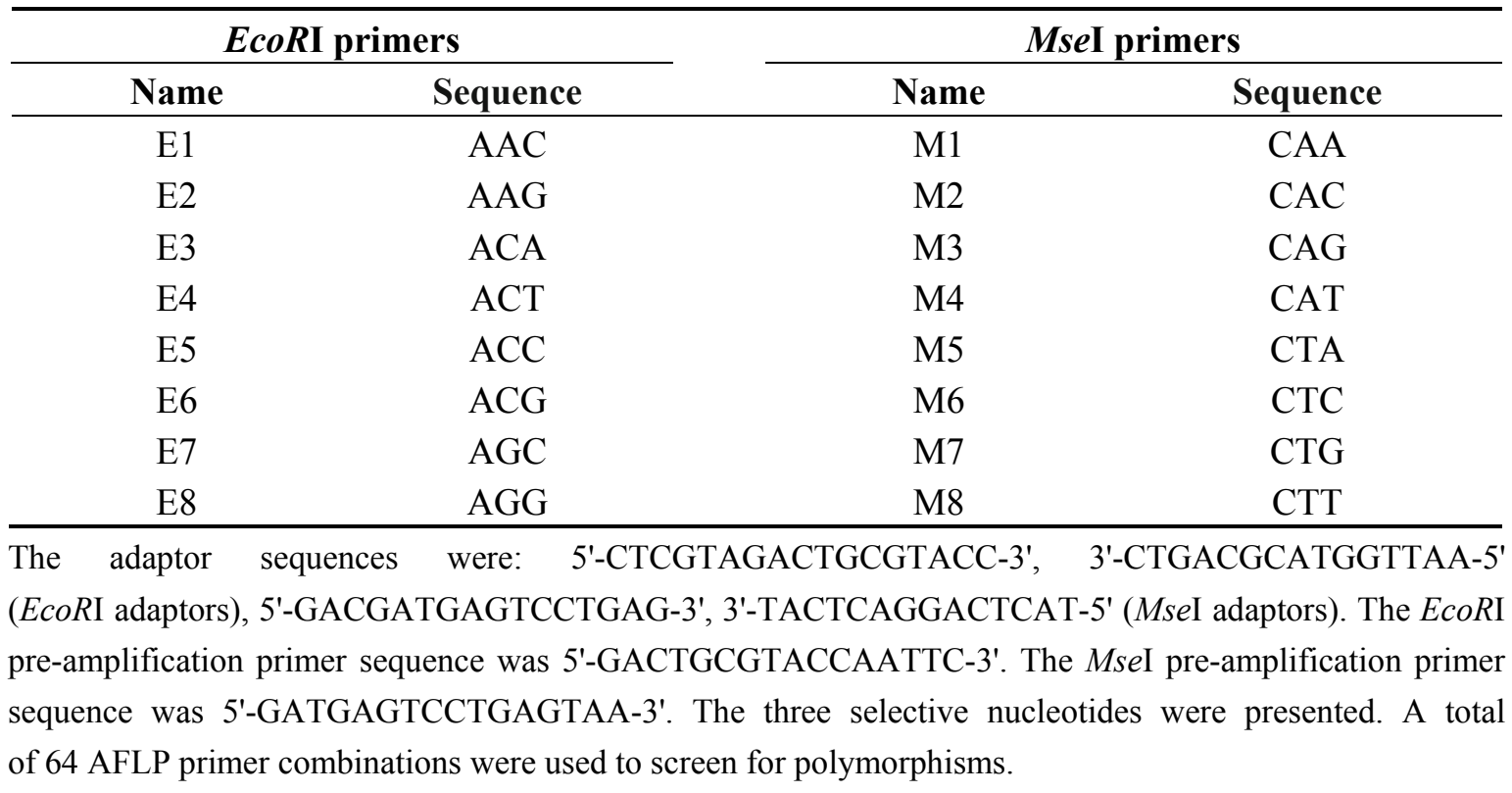

Table 3. Primer sequences used in the inter-simple sequence repeat analysis.

\begin{tabular}{ccc}
\hline Name & Sequence & Annealing temperature \\
\hline UBC808 & $\mathrm{C}(\mathrm{AG})^{8} \mathrm{C}$ & 56.0 \\
UBC811 & $(\mathrm{GA})^{8} \mathrm{C}$ & 43.6 \\
UBC830 & $(\mathrm{TG})^{8} \mathrm{G}$ & 50.0 \\
UBC834 & $(\mathrm{AG})^{8} \mathrm{YT}$ & 56.0 \\
UBC835 & $(\mathrm{AG})^{8} \mathrm{YC}$ & 43.6 \\
UBC840 & $(\mathrm{GA})^{8} \mathrm{YT}$ & 56.0 \\
UBC842 & $(\mathrm{GA})^{8} \mathrm{YG}$ & 54.7 \\
UBC853 & $(\mathrm{TC})^{8} \mathrm{RT}$ & 46.3 \\
UBC860 & $(\mathrm{TG})^{8} \mathrm{RA}$ & 56.0 \\
UBC866 & $\mathrm{CT}(\mathrm{CCT})^{5} \mathrm{C}$ & 52.6 \\
UBC867 & $(\mathrm{GGC})^{6}$ & 41.4 \\
UBC868 & $(\mathrm{GAA})^{6}$ & 46.3 \\
UBC873 & $(\mathrm{GACA})^{4}$ & 50.0 \\
UBC880 & $(\mathrm{GGAGA})^{3}$ & 50.0 \\
UBC881 & $(\mathrm{GGGGT})^{3}$ & 50.0 \\
UBC886 & $\mathrm{VDV}(\mathrm{CT})^{7}$ & 52.6 \\
\hline
\end{tabular}

$R=(A, G) ; Y=(C, T) ; D=(A, G, T) ; V=(A, C, G)$. 
Table 4. Polymorphic markers detected and their segregation ratios.

\begin{tabular}{|c|c|c|c|c|c|c|c|c|c|}
\hline Marker type $^{a}$ & $\begin{array}{l}\text { No. of primer } \\
\text { combinations }{ }^{b}\end{array}$ & $\begin{array}{c}\text { No. of polymorphic } \\
\text { markers }\end{array}$ & $\begin{array}{l}\text { Lmxll } \\
(1: 1)^{c}\end{array}$ & $\begin{array}{l}\text { Nnxnp } \\
(1: 1)^{d}\end{array}$ & $\begin{array}{l}\text { Hkxhk } \\
(3: 1)^{\mathrm{e}}\end{array}$ & $\begin{array}{l}\text { Hkxhk } \\
(1: 2: 1)^{f}\end{array}$ & $\begin{array}{c}\text { Egxef } \\
(1: 1: 1: 1)^{g}\end{array}$ & $\begin{array}{c}\text { Abxed } \\
(1: 1: 1: 1)^{h}\end{array}$ & $\begin{array}{c}\text { Distorted markers } \\
(p<0.05) \\
\end{array}$ \\
\hline SRAP & 131 & 1604 & 305 & 326 & 382 & 18 & 13 & 8 & 552 \\
\hline AFLP & 18 & 295 & 141 & 108 & 22 & 0 & 0 & 0 & 24 \\
\hline ISSR & 16 & 111 & 27 & 23 & 31 & 0 & 0 & 0 & 30 \\
\hline SSR & 17 & 132 & 33 & 42 & 26 & 6 & 7 & 1 & 17 \\
\hline Total & 182 & 2142 & 506 & 499 & 461 & 24 & 20 & 9 & 623 \\
\hline
\end{tabular}

a SRAP sequence-related amplified polymorphism, $A F L P$ amplified fragment length polymorphism, ISSR inter-simple sequence repeat, SSR simple sequence repeat;

${ }^{\mathrm{b}}$ No. of primer combinations primer combination for SRAP, AFLP and SSR, single primer for ISSR; ${ }^{\mathrm{c}}$ lmxll (1:1) lmxll marker, present in the female parent only, segregating 1:1 (11:1m) in the progeny; ${ }^{\mathrm{d}}$ nnxnp (1:1) nnxnp marker, present in the male parent only, segregating 1:1 (nn:np) in the progeny; ${ }^{\mathrm{e}} h k x h k(3: 1)$ hkxhk marker, heterozygous in both parents, segregating 3:1 (hh+hk+h-:kk) in the progeny; ${ }^{\mathrm{f}} h k x h k(1: 2: 1) \mathrm{hkxhk}$ marker, heterozygous in both parents, segregating 1:2:1 (hh:hk:kk) in the progeny; ${ }^{\mathrm{g}}$ egxef $(1: 1: 1: 1)$ egxef marker, heterozygous in both parents, segregating $1: 1: 1: 1$ (ee:ef:eg:fg) in the progeny; ${ }^{\mathrm{h}}$ abxcd $(1: 1: 1: 1)$ abxcd marker, heterozygous in both parents, segregating 1:1:1:1 (ac:ad:bc:bd) in the progeny.

Table 5. Linkage group (LG), markers mapped and marker density for the genetic linkage map of DZ0901 population.

\begin{tabular}{cccccccc}
\hline Linkage group & Length (cM) & No. of markers & SRAPs & AFLPs & ISSRs & SSRs & Mean distance (cM) \\
\hline LG1 & 153.0 & 106 & 70 & 7 & 0 & 29 & 1.5 \\
LG2 & 194.0 & 77 & 9 & 65 & 0 & 3 & 2.6 \\
LG3 & 189.5 & 76 & 74 & 0 & 2 & 0 & 2.5 \\
LG4 & 96.5 & 65 & 64 & 0 & 0 & 1 & 1.5 \\
LG5 & 60.5 & 49 & 37 & 0 & 5 & 7 & 1.3 \\
LG6 & 123.5 & 45 & 0 & 45 & 0 & 0 & 2.8 \\
LG7 & 82.8 & 37 & 37 & 0 & 0 & 0 & 2.3 \\
LG8 & 72.9 & 26 & 25 & 0 & 0 & 1 & 2.9 \\
LG9 & 88.9 & 25 & 12 & 0 & 0 & 13 & 3.7 \\
LG10 & 66.2 & 25 & 25 & 0 & 0 & 0 & 2.8 \\
LG11 & 70.7 & 21 & 20 & 0 & 1 & 0 & 3.5 \\
\hline
\end{tabular}


Table 5. Cont.

\begin{tabular}{cccccccc}
\hline Linkage group & Length (cM) & No. of markers & SRAPs & AFLPs & ISSRs & SSRs & Mean distance (cM) \\
\hline LG12 & 92.9 & 21 & 21 & 0 & 0 & 0 & 4.7 \\
LG13 & 67.1 & 19 & 18 & 0 & 1 & 0 & 3.7 \\
LG14 & 60.7 & 13 & 13 & 0 & 0 & 0 & 5.1 \\
LG15 & 57.3 & 12 & 11 & 0 & 1 & 0 & 5.2 \\
LG16 & 54.3 & 12 & 12 & 0 & 0 & 0 & 4.9 \\
LG17 & 89.6 & 11 & 10 & 0 & 1 & 0 & 9.0 \\
LG18 & 106.5 & 10 & 10 & 0 & 0 & 0 & 11.8 \\
LG19 & 41.8 & 10 & 10 & 0 & 0 & 0 & 4.6 \\
LG20 & 19.9 & 10 & 6 & 0 & 4 & 0 & 2.2 \\
LG21 & 63.5 & 8 & 7 & 0 & 1 & 0 & 9.1 \\
LG22 & 92.8 & 8 & 8 & 0 & 0 & 0 & 13.3 \\
LG23 & 49.1 & 8 & 8 & 0 & 0 & 0 & 7.0 \\
LG24 & 92.0 & 7 & 3 & 0 & 2 & 2 & 15.3 \\
LG25 & 47.0 & 5 & 5 & 0 & 0 & 0 & 11.8 \\
Total & 2133.0 & 706 & 515 & 117 & 18 & 56 & 3.1 \\
\hline
\end{tabular}

SRAP sequence-related amplified polymorphism; AFLP amplified fragment length polymorphism; ISSR inter-simple sequence repeat; SSR simple sequence repeat.

Figure 1. A genetic linkage map of Eucommia ulmoides based on DZ0901 population and quantitative trait loci (QTLs) for growth-related traits. Map units (cM) shown on the left of each linkage group (LG) were calculated by Kosambi mapping function. Markers are on the right side of the linkage groups. The markers are named with the code referring to the corresponding primer or primer combination (see Tables 1-3), followed by the estimated size of the DNA fragment in nucleotides. The map contains a total of 706 molecular markers, 515 SRAP markers, 117 AFLP markers, 18 ISSR markers and 56 SSR markers. The map spans 25 linkage groups (LG1-LG25) and covers a total genetic distance of $2133 \mathrm{cM}$. 1-LOD and 2-LOD support intervals of each QTL are marked by thick and thin bars, respectively. Red bars represent QTLs for height. Green bars represent QTLs for basal diameter. Blank bars represent QTLs for the traits measured in 2010. Solid bars represent QTLs for the traits measured in 2011. Bars filled with one-sided hatch lines represent QTLs for the traits measured in 2012. Bars filled with two-sided hatch lines represent QTLs for the traits measured in 2013. 
LG1

\begin{tabular}{|c|c|c|c|}
\hline 0.07 & DZ158-320 & & \\
\hline $7.8-$ & em 14 me6-50c & & \\
\hline $9.2-$ & DZ158-270 & & \\
\hline 9.4 & - DZ36-170 & & \\
\hline $12.6-$ & DZ158-240 & & \\
\hline $18.6-$ & - em 1 me4-140c & & \\
\hline 19.4 & - em 18me11-1600c & & \\
\hline $20.0-$ & DZ36-250 & & \\
\hline $20.5-$ & - em 9me15-390 & & \\
\hline & DZ38-121c & & \\
\hline 23.5 & - DZ36-172 & & \\
\hline & - em6me8-330c & & \\
\hline & DZ36-180 & & \\
\hline 28.9 & - em 9me5-300 & & \\
\hline 29.0 & $\begin{array}{l}\text { - em8me10-230c } \\
\text { - em 45me6-620 }\end{array}$ & & E3M4-330 \\
\hline & $\begin{array}{l}\text { em45me6-620 } \\
\text { DZ159-260 }\end{array}$ & $\begin{array}{r}0.0 \\
20.27\end{array}$ & E1M1-400 \\
\hline 32.0 & $\begin{array}{l}\text { DZ159-260 } \\
\text { em25me28-110c }\end{array}$ & 30.0 & \\
\hline & $\begin{array}{l}\text { em } 25 m e 28-110 c \\
\text { em } 26 m e 13-100\end{array}$ & $\begin{array}{l}30.0 \\
33.9\end{array}$ & [E1M1-300 \\
\hline & - em26me13-100 & $\left.\begin{array}{l}33.9 \\
34.5\end{array}\right]$ & E1M1-300 \\
\hline & em 1 me $4-1500 c$ & $\begin{array}{l}34.5 \\
39.9\end{array}$ & $\begin{array}{l}\text { E7M6-520 } \\
\text { E8M8-160 }\end{array}$ \\
\hline & $\begin{array}{l}\text { em56me4-50 } \\
D Z 36-205\end{array}$ & $\begin{array}{l}39.9 \\
43.0\end{array}$ & E8M8-160 \\
\hline & DZ36-205 & $\begin{array}{l}43.0 \\
45.8\end{array}$ & E3M4-300 \\
\hline & $D Z 165-301 c$ & 45.8 & - Е8M8-60 \\
\hline & em56me4-100 & 51.9 & E4M8-480 \\
\hline & DZ159-150 & $53.0-$ & E6M6-44O \\
\hline 41.6 & em 4 me3-2350 & $58.0-$ & - E7M8-380 \\
\hline & em7me $12-270 c$ & 58.9 & E4M8-470 \\
\hline & em 4 me3-2300 & $59.4-$ & E7M4-60 \\
\hline 42.7 & em9me15-650 & 62.4 & - E5M4-650 \\
\hline 42.8 & $-D Z 159-145$ & 63.0 & E7M4-160 \\
\hline & - DZ38-140 & 66.3 & E5M4-700 \\
\hline 43.9 & em4me4-80 & 68.2 & - E5M4-600 \\
\hline & em4me3-2400 & 69.3 & E7M8-500 \\
\hline & DZ32-210 & 73.1 & E1M5-180 \\
\hline 47.2 & em 1 me26-110 & 74.4 & E5M4-560 \\
\hline & DZ159-320 & 75.3 & - E7M6-300 \\
\hline & - em4me3-1000 & 78.3 & - E7M4-570 \\
\hline 49.5 & - em2me13-70 & 79.0 & - E4M8-250 \\
\hline 50.1 & - em11me12-220 & 79.8 & E6M4-50 \\
\hline 50.5 & - DZ159-170 & $83.9-1-2-2-$ & E1M2-600 \\
\hline 51.6 & em9me15-1300 & 85.27 & - E7M6-360 \\
\hline 52.8 & em 13 me4-190c & 86.7 & - E5M4-680 \\
\hline 53.4 & - DZ126-301c & 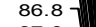 & E7M6-400 \\
\hline 56.0 & $-D Z 4-141 c$ & $87.0-$ & E6M4-350 \\
\hline 56.1 & em 15 me $10-60$ & $90.0-$ & E7M6-50 \\
\hline 57.0 & E8M8-115 & $92.1]$ & E7M6-540 \\
\hline 57.1 & Lem53me13-320 & $92.5-7-1-1-2$ & \\
\hline & -em9me5-310 & $95.0]$ & {$\left[\begin{array}{l}\text { E1M5-250 } \\
\text { E8M5-480 }\end{array}\right.$} \\
\hline 58.8 & - DZ159-180 & $97.6]$ & \\
\hline 58.9 & - em 1me26-255 & $98.5-$ & E6M4-80 \\
\hline & em8me10-480 & 100.4 & E6M4-210 \\
\hline 61.8 & em9me11-140 & $100.6-$ & | E7M6-610 \\
\hline & em 1me14-900 & 102.5 & Е8M5-440 \\
\hline $63.1-$ & E3M6-125 & $103.0-$ & E3M6-600 \\
\hline 63.2 & DZ159-280 & $104.6 \sqrt{ }$ & W E6M4-130 \\
\hline 63.4 & em8me11-500 & 106.67 & - DZ32-320 \\
\hline 64.1 & em2me13-200 & 107.3 & - Е8M8-440 \\
\hline & DZ159-205 & 107.4 & E EMM4-320 \\
\hline 67.9 & em9me15-1100 & $107.6 \mathrm{~J}$ & L E1M1-250 \\
\hline 68.4 & E1M2-60 & 112.3 & - E5M4-530 \\
\hline & em3me9-350 & 113.7 & L- E7M8-240 \\
\hline 70.0 & em5me14-150 & $114.1 \mathrm{~J}$ & L DZ32-310 \\
\hline & em5me14-330 & $114.9-1$ & LE5M4-500 \\
\hline 70.9 & em $1 \mathrm{me} 26-2400$ & $115.2 \mathrm{~J}$ & $\begin{array}{l}\text { E E1M8-100 } \\
\text { E1M2-480 }\end{array}$ \\
\hline & em49me3-150 & 116.1 - & {$\left[\begin{array}{l}\text { E1M2-480 } \\
\text { E7M4-350 }\end{array}\right.$} \\
\hline & em 1 me6-1600 & $116.9-$ & {$\left[\begin{array}{l}\text { E7M4-350 } \\
\text { E8M5-320 }\end{array}\right.$} \\
\hline & $\begin{array}{l}\text { em13me4-620 } \\
\text { DZ140-341c }\end{array}$ & $\begin{array}{l}119.7 \\
120.8\end{array}$ & L E $\begin{array}{l}\text { EM5-320 } \\
\text { DZ32-420 }\end{array}$ \\
\hline & $\begin{array}{l}\text { DZ140-341c } \\
\text { DZ159-201c }\end{array}$ & 121.6 & E8M8-530 \\
\hline & $\mathrm{E} 2 \mathrm{M} 6-2 \mathrm{O} 4$ & 123.1 & E8M8-400 \\
\hline 74.2 & em60me6-205 & 125.2 & E7M8-350 \\
\hline 74.3 & em 1 me14-550 & 127.7 & E5M4-370 \\
\hline & E5M4-215 & 128.6 & - E7M4-600 \\
\hline 76.3 & E8M8-125 & 131.0 & - E7M4-280 \\
\hline 78.2 & em4me11-120 & 131.1 & E4M2-500 \\
\hline & em5me8-710c & 132.0 & - E8M5-470 \\
\hline 80.0 & em 13 me $5-200$ & 132.3 & -em44me9-115 \\
\hline 80.2 & DZ159-195 & 136.8 & - E6M4-110 \\
\hline & em41me19-175 & 136.9 & - em 1 me10-2200 \\
\hline 81.7 & em8me11-800 & $142.0-$ & em 10me6-650 \\
\hline 82.6 & em2me2-70 & $143.9-$ & - E7M4-300 \\
\hline 83.6 & E1M8-245 & 148.0 & - E5M4-450 \\
\hline & em11me14-59o & 148.1 & - E6M4-280 \\
\hline 85.6 & em 15me16-290 & 151.6 & - em $32 m e 19-215 c$ \\
\hline 87.1 & em 10me6-2000 & $156.1-$ & E7M6-240 \\
\hline & em6me14-260c & $161.3-1$ & - E6M4-150 \\
\hline & em 1 me9-200c & $165.8-$ & $\begin{array}{l}\text { em 1me1-2200 } \\
\text { em 1me4-2300 }\end{array}$ \\
\hline & - em8me26-1300c & $170.5-1$ & $\begin{array}{l}\text { em } 1 \mathrm{me} 4-2300 \\
\text { em } 1 \mathrm{me} 2-2200\end{array}$ \\
\hline 91.8 & - em8me11-120 & 176.1 - $] \mid$ & $\mathbb{L} \mathbb{L}_{\text {E1M5-400 }}^{\text {em } 1 \text { me2-2200 }}$ \\
\hline & $\begin{array}{l}\text { em } 4 m e 7-390 \\
\text { em } 13 m e 4-390\end{array}$ & $\begin{array}{l}181.2-1 \\
188.1\end{array}$ & $\mathbb{L}_{\text {em } 1 \mathrm{me}-2100}$ \\
\hline $\begin{array}{l}96.7 \\
98.2\end{array}$ & em 13me4-290c & $194.0\rfloor$ & L em 1me9-2300 \\
\hline
\end{tabular}

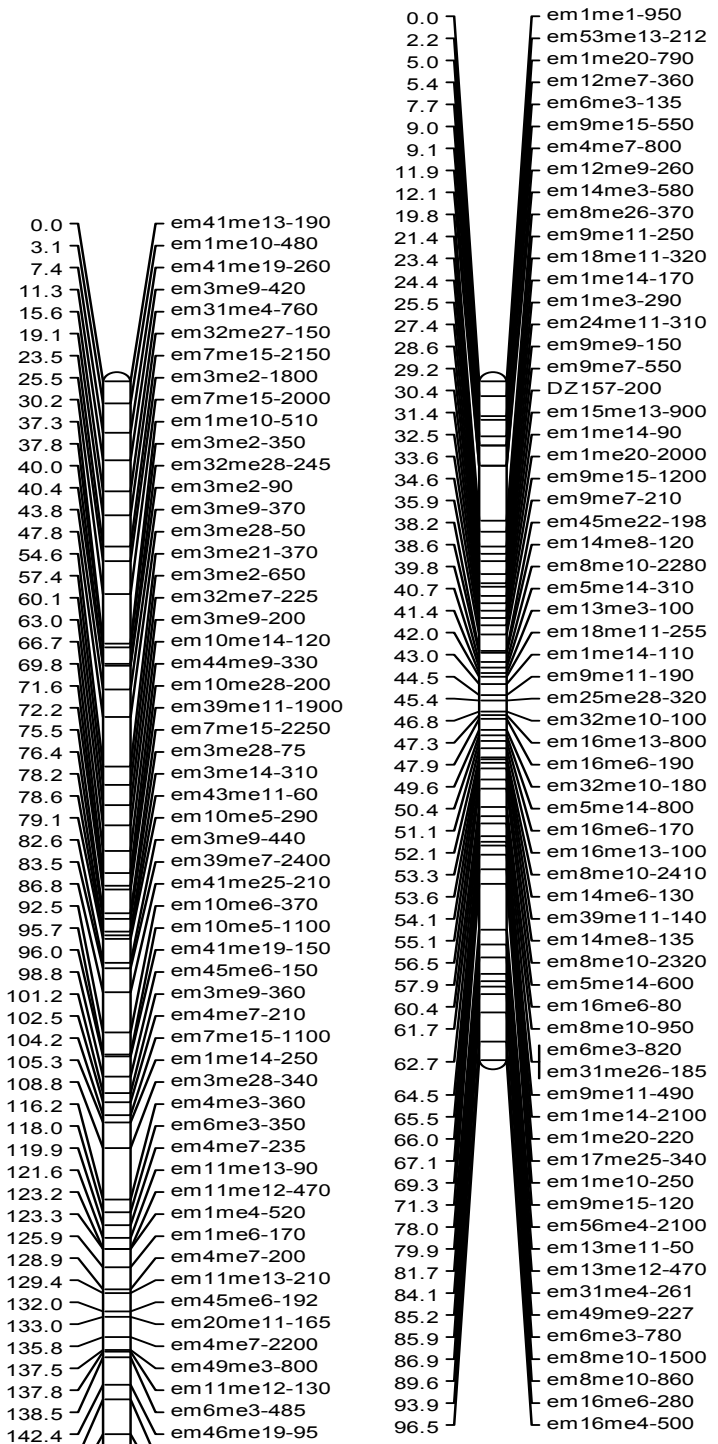


LG5

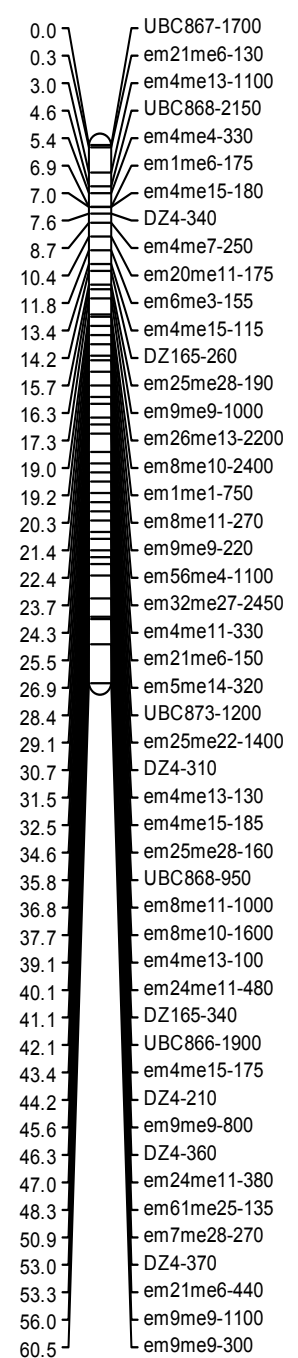

LG6

LG7

0.07 em3me12-180

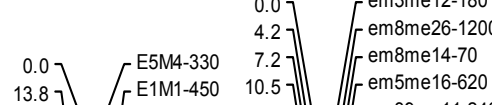

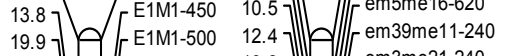

23.8 ] [ [ E7M6-260 19.8 .

31.2]

$31.9]\left[\begin{array}{lll}E 7 M 6-150 & 25.1\end{array}\right]$ E em8me20-105

33.6 ] - E4M2-330 25.3 $]$ [ [ [ em43me11-190

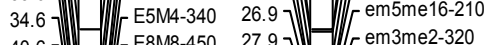

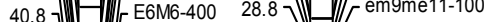

$41.3]\left[\begin{array}{ll}E 5 M 4-310 & 29.6\end{array}\right]$ Em5me16-550

40.8 = ETM6-430 30.2 - em Ime6-2000

46.8 $=$ E8M8-520 $33.0-$

50.8 -

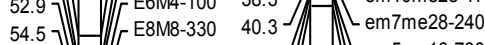

$57.9]=\left[\begin{array}{ccc}E S M 4-240 & 42.0\end{array}\right] \quad[-$ em5me16-700

04.5]

$65.0]=($ E5M4-220 51.9$] / 2[$ em43me11-140

$67.8]\left[\begin{array}{cc}\text { E7M8-400 } & 54.6 \\ \text { E1M8-330 } & 56.8\end{array}\right]=\left[\begin{array}{l}\text { em5me16-330 } \\ \text { em8me9-110 }\end{array}\right.$

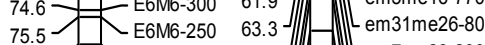

78.4 _

80.7 - ESM4-90 68.1 F

83.9

$\left.\begin{array}{l}86.8 \\ 87.2\end{array}\right]=\left[\begin{array}{ll}E 6 M 4-440 & 73.0 \\ E 1 M 2-470 & 76.9\end{array}\right]\left[\begin{array}{l}\text { em8me26-500 } \\ \text { em41me13-250 } \\ \text { em5me16-380 }\end{array}\right.$

$\left.\begin{array}{l}87.2 \\ 89.5\end{array}\right]\left[\begin{array}{cc}-E 1 M 2-470 & 76.9 \\ \text { E8M5-430 } & 78.8\end{array}\right]$

$0.0 \gamma^{\text {em26me29-265 }}$

em em 4.9 me9-225

14.9 $]-\left[\begin{array}{l}\text { em8me26-50 } \\ \text { em28me25-160 }\end{array}\right.$

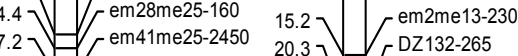

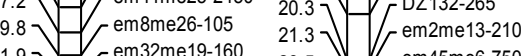

Em2me13-1500

27.4 -

$32.8]=\left[\begin{array}{l}\text { em } 45 m e 6-750 \\ \text { em } 45 \text { me6-2200 }\end{array}\right.$

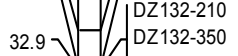

- $\underbrace{\text { DZ132-335 }}_{\text {em45me6-265 }}$

36.9

46.0 DZ132-260

49.3 DZ126-440

53.2 - em28me25-350

55.6- DZ132-212

em3me14-370

7.6.

- em9me15-500 60.5$)={ }^{D Z 132-400}$

em9me1-700

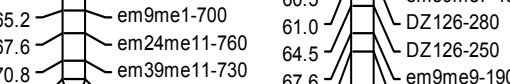

$67.6]-\left[\begin{array}{l}\text { em9me9-190 } \\ \text { DZ126-270 }\end{array}\right.$

69.8 $\begin{aligned} & \text { DZ126-270 } \\ & \text { em9me9-170 }\end{aligned}$

88.9- em13me4-180c $50.0]\left[\begin{array}{lll}0.0 \\ \text { ESM-300 } & 36.4\end{array}\right.$

70.8 - E6M4-120 57.8

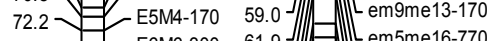

em40me6-620

92.4 [ E5M4-120

96.0 - E8M8-110

106.4

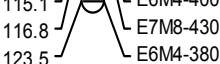

LG10

LG11

LG12

Figure 1. Cont.

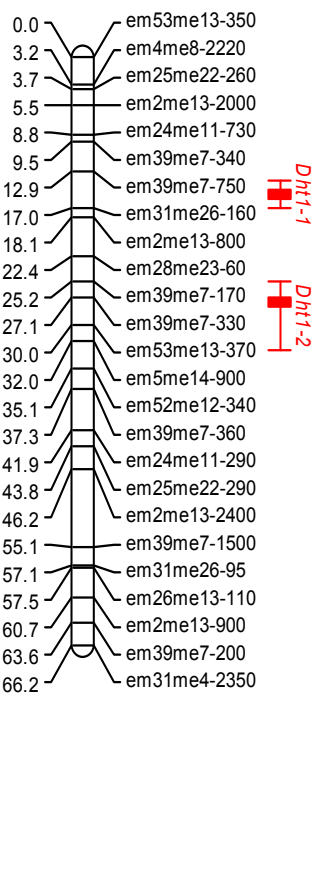

0.0 em4me4-90 2.8 em4me7-230 em4me13-395 - em8me9-80

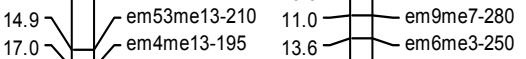
18.6 $=$ em53me13-240

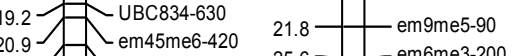
23.8 - em6me3-2450 25.6- em6me3-200 26.3 em9me1-230 35.0- em25me22-180 $37.5-$ em21me6-660 40.1 em $45 \mathrm{me6}-190$ $44.1-$ em20me22-175 31.0 em6me3-550 48.7- em5me8-245 $52.3-$ em 20me22-15

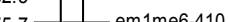
\begin{tabular}{l|l} 
em $9 \mathrm{me} 7-230$ \\
43.0 & em $9 \mathrm{me} 15-450$
\end{tabular}

63.968.5 - em1me9-2200 $46.1-$ em 1 me5-210 $51.2-$ em6me3-248 63.1 - em8me9-270 $65.9-$ em8me6-520 - em1me6-330

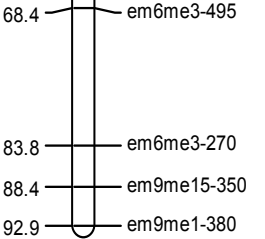


Figure 1. Cont.

LG13

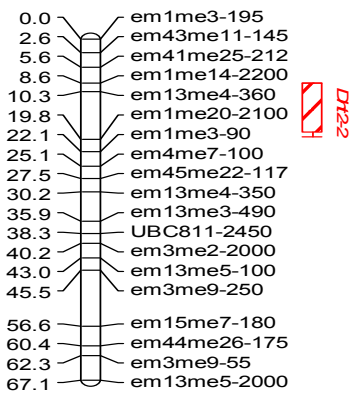

LG18

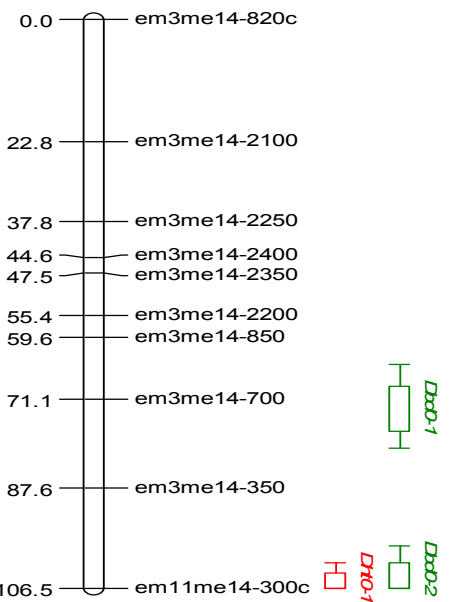

LG22

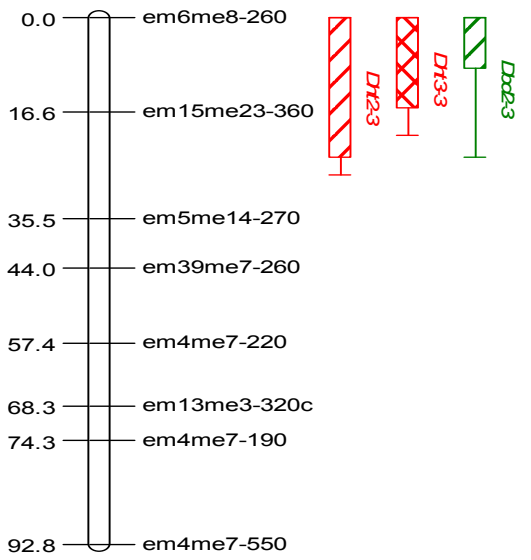

LG15
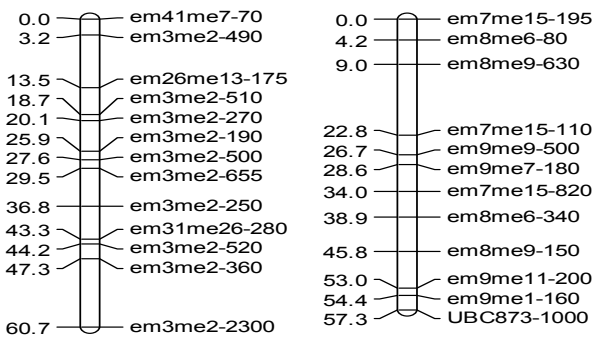

LG16

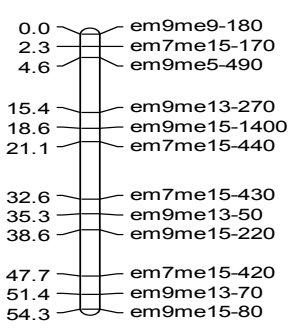

LG17

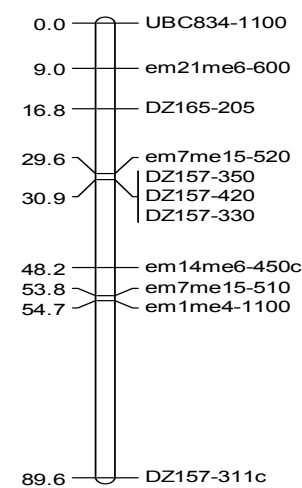

LG19

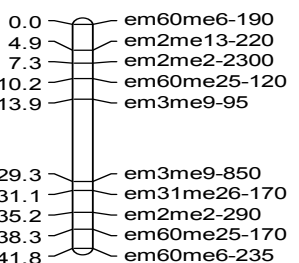

LG23

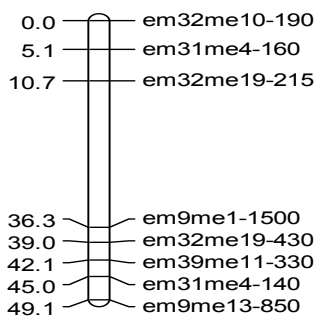

LG20

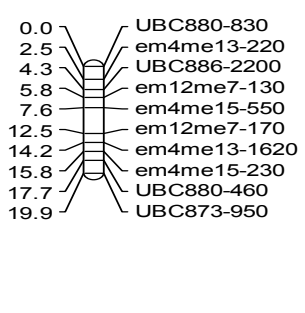

LG24

LG25

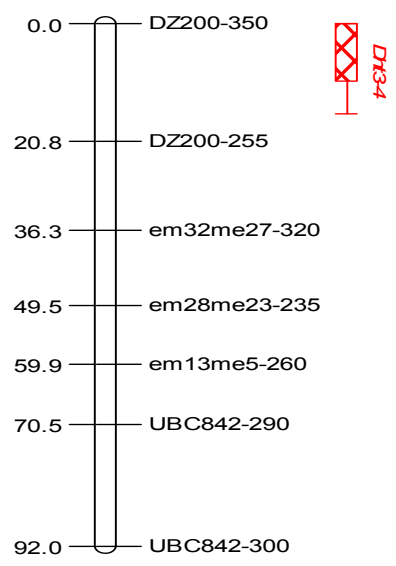

\subsection{Growth Traits and QTL Analysis}

A high degree of genetic variation was found for height and basal diameter (Table 6). Figure 2 showed the frequency distributions of these traits. Pearson correlation analyses showed significant correlations between height and basal diameter and moderate weak correlations over years (Table 7). 
Table 6. Mean, standard deviation (SD), range and coefficient of variation (CV) for the growth traits.

\begin{tabular}{cccccc}
\hline Trait & Mean & SD & Minimum & Maximum & CV (\%) \\
\hline Height 2010 $(\mathrm{cm})$ & 39.3 & 16.8 & 9.0 & 85.0 & 42.8 \\
Height 2011 $(\mathrm{cm})$ & 138.7 & 36.6 & 50.0 & 216.0 & 26.4 \\
Height 2012 $(\mathrm{cm})$ & 224.8 & 48.4 & 120.0 & 310.0 & 21.5 \\
Height 2013 $(\mathrm{cm})$ & 332.0 & 74.4 & 170.0 & 480.0 & 22.4 \\
Basal diameter 2010 (mm) & 4.9 & 1.7 & 1.3 & 9.4 & 34.0 \\
Basal diameter 2011 (mm) & 11.9 & 3.1 & 3.9 & 19.6 & 25.8 \\
Basal diameter 2012 (mm) & 19.2 & 4.6 & 10.0 & 30.4 & 24.0 \\
Basal diameter 2013 (mm) & 22.4 & 6.1 & 10.4 & 38.3 & 27.2 \\
\hline
\end{tabular}

Table 7. Pearson correlation coefficients between the growth traits.

\begin{tabular}{|c|c|c|c|c|c|c|c|}
\hline Traits & $\begin{array}{c}\text { Height } \\
2011 \\
\end{array}$ & $\begin{array}{c}\text { Height } \\
2012 \\
\end{array}$ & $\begin{array}{l}\text { Height } \\
2013 \\
\end{array}$ & $\begin{array}{c}\text { Basal } \\
\text { diameter } 2010 \\
\end{array}$ & $\begin{array}{c}\text { Basal } \\
\text { diameter } 2011 \\
\end{array}$ & $\begin{array}{c}\text { Basal } \\
\text { diameter } 2012\end{array}$ & $\begin{array}{c}\text { Basal } \\
2 \text { diameter } 2013 \\
\end{array}$ \\
\hline Height 2010 & $0.52 *$ & -0.04 & -0.02 & $0.80 *$ & $0.51 *$ & 0.02 & 0.03 \\
\hline Height 2011 & & 0.04 & 0.02 & $0.51 *$ & $0.83 *$ & 0.03 & 0.15 \\
\hline Height 2012 & & & $0.70 *$ & -0.02 & 0.03 & $0.66 *$ & $0.75 *$ \\
\hline Height 2013 & & & & 0.05 & -0.04 & $0.64 *$ & $0.72 *$ \\
\hline Basal diameter 2010 & & & & & $0.47 *$ & 0.01 & 0.04 \\
\hline Basal diameter 2011 & & & & & & 0.10 & 0.10 \\
\hline Basal diameter 2012 & & & & & & & $0.92 *$ \\
\hline
\end{tabular}

$$
* p<0.01
$$

Figure 2. Frequency distributions of growth traits for DZ0901 population.

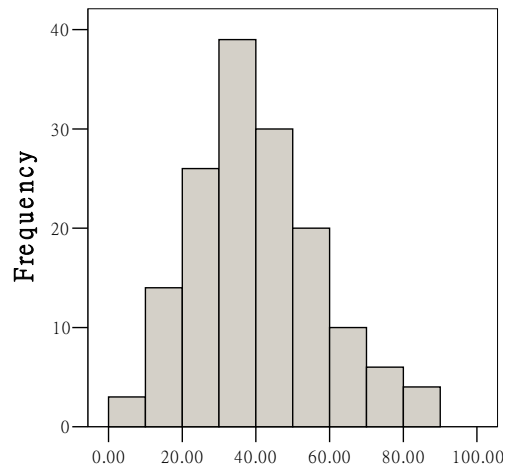

Height in $2010(\mathrm{~cm})$

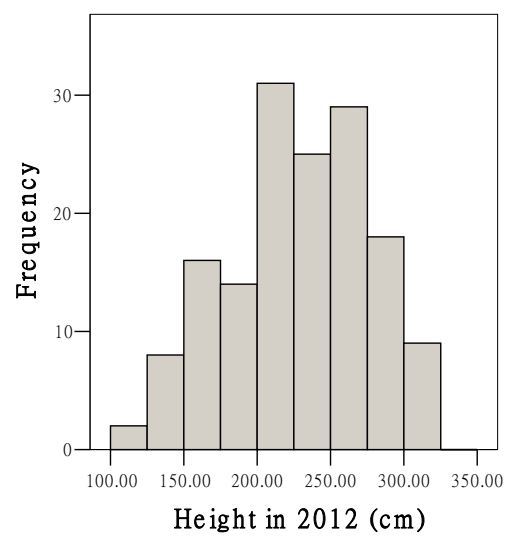

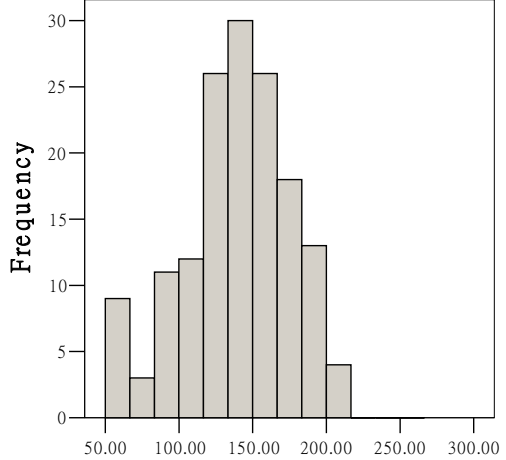

Height in $2011(\mathrm{~cm})$

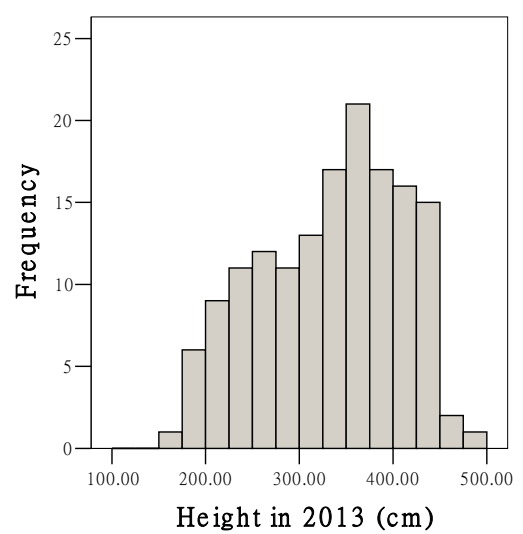


Figure 2. Cont.
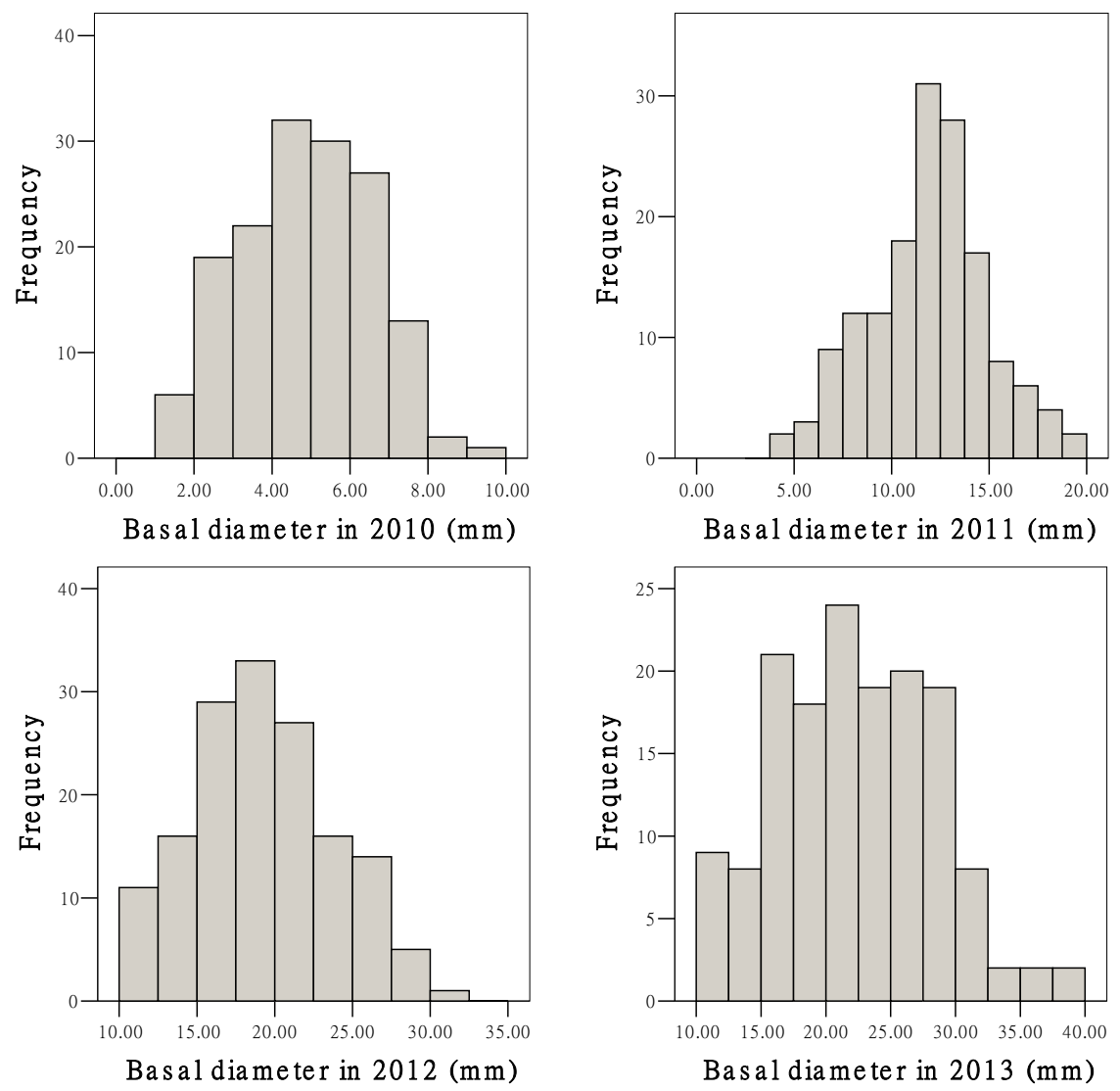

The genetic linkage map of DZ0901 was used to search for putative QTLs (Table 8 and Figure 1). Eleven height QTLs were detected. In 2010, one height QTL was located on LG18 and explained $17.1 \%$ of the phenotypic variation. In 2011, three additional height QTLs were located on LG10, LG10 and LG12, and explained $29.7 \%, 27.7 \%$ and $22.8 \%$ of the phenotypic variation, respectively. In 2012 , three height QTLs were located on LG9, LG13 and LG22, and explained 12.6\%, 12.4\% and 33.3\% of the phenotypic variation, respectively. In 2013, two height QTLs identified at similar genomic regions as the height QTLs in 2012 were located on LG9 and LG22, and explained 13.5\% and 25.3\% of the phenotypic variation, respectively. Other two height QTLs in 2013 were located on LG21 and LG24, and explained $26.6 \%$ and $27.1 \%$ of the phenotypic variation, respectively. Four basal diameter QTLs were identified at similar genomic regions as the height QTLs. In 2010, one basal diameter QTLs was located on LG18 and explained 13.4\% of the phenotypic variation. In 2011, one basal diameter QTLs was located on LG12 and explained $20.2 \%$ of the phenotypic variation. In 2012, two basal diameter QTLs were located on LG21 and LG22, and explained $25.1 \%$ and $21.4 \%$ of the phenotypic variation, respectively. Three additional basal diameter QTLs were detected on LG18, LG1 and LG1, and explained $29.8 \%, 17.7 \%$ and $16.8 \%$ of the phenotypic variation, respectively. Four of the 18 QTLs were significant, and they were Dht0-1, Dht1-1, Dht1-2 and Dbd0-2. Other QTLs were not significant, but they had a LOD score greater than 3.0. Flanking markers and QTLs supported by Kruskal-Wallis nonparametric test were indicated in Table 8. 
Table 8. QTLs of growth traits detected in the DZ0901 population.

\begin{tabular}{|c|c|c|c|c|c|c|}
\hline QTL $^{\text {a }}$ & Linkage group & Peak position (cM) ${ }^{b}$ & LOD peak $^{c}$ & Marker $^{d}$ & $\%$ Var.expl. ${ }^{\mathrm{e}}$ & $\mathbf{K W}^{\mathrm{f}}$ \\
\hline \multicolumn{7}{|c|}{ Height 2010} \\
\hline Dht0-1 & LG18 & 106.5 & $7.8 * *$ & em $11 \mathrm{me} 14-300 \mathrm{c}$ & 17.1 & $* * * *$ \\
\hline \multicolumn{7}{|c|}{ Height 2011} \\
\hline Dht1-1 & LG10 & 14.9 & $4.4 * *$ & em39me7-750 & 29.7 & - \\
\hline Dht $1-2$ & LG10 & 27.1 & $4.4 * *$ & em39me7-330 & 27.7 & $*$ \\
\hline Dht1-3 & LG12 & 40.1 & 3.1 & em9me7-230 & 22.8 & - \\
\hline \multicolumn{7}{|c|}{ Height 2012} \\
\hline Dht2-1 & LG9 & 62.0 & 3.2 & DZ126-280 & 12.6 & - \\
\hline Dht2-2 & LG13 & 14.3 & 3.1 & em13me4-360 & 12.4 & $* * * *$ \\
\hline Dht2-3 & LG22 & 14.0 & 3.3 & em15me23-360 & 33.3 & - \\
\hline \multicolumn{7}{|c|}{ Height 2013} \\
\hline Dht3-1 & LG9 & 62.0 & 3.9 & DZ126-280 & 13.5 & - \\
\hline Dht3-2 & LG21 & 57.4 & 3.3 & UBC881-820 & 26.6 & - \\
\hline Dht3-3 & LG22 & 0.0 & 3.8 & em6me8-260 & 25.3 & - \\
\hline Dht3-4 & LG24 & 0.0 & 4.3 & DZ200-350 & 27.1 & - \\
\hline \multicolumn{7}{|c|}{ Basal diameter 2010} \\
\hline Dbd0-1 & LG18 & 72.1 & 3.8 & em3me14-700 & 29.8 & $* *$ \\
\hline$D b d 0-2$ & LG18 & 106.5 & $4.7 * *$ & em $11 \mathrm{me} 14-300 \mathrm{c}$ & 13.4 & $* * * *$ \\
\hline \multicolumn{7}{|c|}{ Basal diameter 2011} \\
\hline$D b d 1-1$ & LG12 & 40.1 & 3.0 & em9me7-230 & 20.2 & - \\
\hline \multicolumn{7}{|c|}{ Basal diameter 2012} \\
\hline$D b d 2-1$ & LG1 & 153.0 & 3.0 & em12me11-300 & 17.7 & - \\
\hline $\operatorname{Dbd2-2}$ & LG21 & 58.4 & 3.2 & em5me7-530 & 25.1 & $* *$ \\
\hline$D b d 2-3$ & LG22 & 0.0 & 3.6 & em6me8-260 & 21.4 & $* *$ \\
\hline \multicolumn{7}{|c|}{ Basal diameter 2013} \\
\hline$D b d 3-1$ & LG1 & 153.0 & 3.0 & em12me11-300 & 16.8 & - \\
\hline $\begin{array}{l}{ }^{\mathrm{a}} \mathrm{Q} \\
(0 \mathrm{f} \\
\text { posi } \\
\text { perr } \\
\text { phe } \\
(* 0\end{array}$ & $\begin{array}{l}\text { named using an } \\
2010,1 \text { for } 2011,2 \\
\text {; }{ }^{\mathrm{c}} \text { LOD peak max } \\
\text { tation tests; }{ }^{\mathrm{d}} \text { Mark } \\
\text { typic variance expl } \\
* * 0.05 ; * * * 0.00\end{array}$ & $\begin{array}{l}\text { breviation of the trait } \\
\text { or 2012, } 3 \text { for } 2013 \text { ) and } \\
\text { mum LOD value; }{ }^{* *} \text { LOD } \\
\text { r marker name nearest to } \\
\text { ined by the QTL; }{ }^{\mathrm{f}} \mathrm{KW} \\
\text { 15). }\end{array}$ & $\begin{array}{l}\text { ht Height, } D b d \\
\text { he QTL number; } \\
\text { value significan } \\
\text { the QTL positio } \\
\text { ruskal-Wallis s }\end{array}$ & $\begin{array}{l}\text { Basal diameter), foll } \\
{ }^{\mathrm{b}} \text { Peak position log of } \\
\text { at } p<0.05 \text { based on } \\
\text { '; }{ }^{\text {e }} \% \text { Var. expl. prop } \\
\text { gnificance level, give }\end{array}$ & $\begin{array}{l}\text { lowed by the year } \\
\text { f odds (LOD) peak } \\
1000 \text { genome-wide } \\
\text { portion of the total } \\
\text { en by the } p \text { values }\end{array}$ & \\
\hline
\end{tabular}

\section{Discussion}

\subsection{Marker Amplification}

SRAP has been recognized as an efficient and useful marker system [12,13,23]. It has several advantages such as simplicity, high throughput, numerous co-dominant markers and easy isolation of DNA fragments for sequencing, and it targets open reading frame regions. In this mapping population, SRAP analysis was an efficient method for generating polymorphic markers. Every primer combination gave at least six polymorphic markers with an average of 12.2 per primer combination. This is comparable to the polymorphism in other tree mapping projects using SRAP analysis [12,13]. 
It is known that AFLP marker produces a larger number of polymorphic fragments than other techniques. In our study, the average number of polymorphic DNA fragments per primer combination was 16.4. This is comparable to the average obtained in other tree mapping projects [9,17], but lower than the average reported for mapping using interspecific crosses [24,25]. As reported on many plant species [24,25], AFLP markers were dominant in this mapping population.

ISSR analysis has been used successfully to construct genetic linkage map of many tree species $[9,10,12]$. In these studies, ISSR markers were highly polymorphic and tended to be evenly distributed throughout genomes. Besides, the ISSR analysis was faster and easier than the AFLP analysis. However, it was less efficient with an average of 6.9 polymorphic markers per primer and had a limited number of primers. Like AFLP marker, ISSR markers were dominant in this mapping population.

SSR markers are typically co-dominant, highly polymorphic and highly reproducible across laboratories. They are also useful for comparing and combining linkage maps from different mapping populations. Furthermore, many SSR markers are transferable across related species [26,27]. Unfortunately, E. ulmoides is the single extant species of the genus Eucommia, and there are fewer available SSR primer combinations. In this study, we used 19 SSR primer combinations. Additional SSR markers are currently being added to better bridge this map with future E. ulmoides maps.

\subsection{Segregation Distortion}

In this study, 29\% of the markers showed segregation distortion. We excluded these markers to obtain a more accurate genetic linkage map because distorted markers can affect the mapping accuracy by overestimating the map distances and causing marker clustering [9,28]. Also, the order of markers on linkage groups may be affected by segregation distortion [29]. We may have lost some information by excluding the distorted markers. However, we obtained a genetic linkage map covering approximately $89 \%$ of the estimated E. ulmoides genome with an average of $3.1 \mathrm{cM}$ between adjacent markers. In a follow-up study, we intend to map these distorted markers using a larger mapping population and co-dominant markers.

Segregation distortion has been reported frequently in woody species. The percentage of markers showing segregation distortion was highly variable: $47 \%$ in spruce [28], 38\% in pear [25], 29\% in citrus [12], 18\% in Salix [8], 9\% in grape [9], 8.5\% in Populus [13] and 1.8\% in peach [30]. Compared to these data, distorted frequency in this study appeared to be intermediate (29\%). Many biological mechanisms have been implicated in causing segregation distortion including divergence of the parental genotypes [13,25,31], chromosome loss [32], genome size differences [31], genetic load and recessive lethal alleles [33], meiotic drive locus [34], and gametic and zygotic selection [35,36]. In the present study, the female parent Xiaoye was a wild genotype from the forest in Henan province. The male parent Qinzhong No.1 was a cultivar produced by controlled breeding, and it was planted in the museum garden of Northwest A\&F University. They differ in traits of growth, phenology, morphology and content of secondary metabolite. Thus, the divergence of the parental genotypes may contribute to the observed segregation distortion. 


\subsection{Genetic Linkage Map}

We constructed a genetic linkage map of E. ulmoides based on the segregation of SRAP, AFLP, ISSR and SSR markers as a first step towards understanding the E. ulmoides genome. The total map distance was $2133 \mathrm{cM}$, and the average map distance between adjacent markers was $3.1 \mathrm{cM}$. The present map covers a significant portion of the E. ulmoides genome, which should provide adequate coverage of the genome to begin QTL analysis. E. ulmoides is a diploid species with $2 n=34$. The number of linkage groups was more than the number of haploid chromosomes of E. ulmoides. The presence of more than 17 linkage groups may be due to some gaps preventing connection between groups belonging to the same chromosome. However, gaps in the genetic linkage maps, resulting in two or more linkage groups per chromosome, are common in tree species even with large numbers of markers $[8,11,13,15]$. In future work, more co-dominant and functional markers are needed to be added to this genetic linkage map in order to fill the gaps, integrate some linkage groups and cover the entire genome.

\subsection{QTL Analysis}

It is often assumed that a quantitative trait exhibits continuous variation because of the interaction of environmental effects and multiple genes of small and cumulative effects. In the present study, we were able to detect QTLs with moderate to large effect for growth-related traits. The estimated magnitude of the individual QTL effect ranged from $12.4 \%-33.3 \%$ of the phenotypic variance. Our results agree with other QTL studies in tree species indicating that growth-related traits may in part be controlled by a few genes with large effect. In an $\mathrm{F}_{2}$ population based on an interspecific cross of Populus, Bradshaw and Stettler [37] reported that effects of single QTL for growth-related traits explained $24 \%-33 \%$ of the phenotypic variation. In an interspecific backcross family of white poplar, Zhang et al. [38] found that four QTLs for stem volume explained 35.8\% of the total phenotypic variance. In a tetraploid hybrid $\mathrm{F}_{2}$ population of Salix, most of the QTL for the different growth-related traits each explained around $12 \%$ of the phenotypic variation, with a few exceptions explaining more than $20 \%$ of the variation [39]. Furthermore, in an intraspecific cross of Salix, 11 QTLs were identified for growth-related traits with each QTL explaining $14 \%-22 \%$ of the phenotypic variance [8].

Four basal diameter QTLs were identified at similar genomic regions as the height QTLs. This was not surprising because of the high correlation coefficient between height and basal diameter. The Pearson correlation coefficients were 0.80 in 2010, 0.83 in 2011, 0.66 in 2012 and 0.72 in 2013, respectively (Table 7). This suggested that height and basal diameter growth in E. ulmoides had common genetic components. The clustering of QTLs controlling highly correlative growth-related traits have been reported in other tree species of Populus [37,38], Salix [8,39], Eucalyptus [18,19,40] and apple [41].

No QTL was consistently expressed over the four years. However, QTL Dht2-1, Dht2-3 and Dbd2-1 in 2012 were identified at similar genomic regions as the QTL Dht3-1, Dht3-3, and Dbd3-1 in 2013, respectively. A similar result of QTL analysis for height and basal diameter in radiata pine was reported by Emebiri et al. [42], who observed that none of the putative QTL positions detected at any one age was strongly expressed at all of the four stages of measurement and that $45 \%$ of putative QTLs 
significant at one age were also detected at a subsequent age. For growth-related traits, QTL instability has been reported frequently in tree species [8,15,37,40,41,43]. Verhaegen et al. [40] did not find the same QTLs over three consecutive years for growth-related traits in hybrid Eucalyptus. In rubber tree, QTLs detected during the summer were different from the QTLs detected during the winter for height and girth growth [15]. To explain this phenomenon, Verhaegen et al. [40] assumed that a set of regulatory genes may differentially control the temporal expression of the genes controlling a trait or that different sets of regulatory factors may be involved during different periods of time. Kenis and Keulemans [41] proposed that genetic control of these traits is largely influenced by environmental factors and probably changes as the tree matures.

To be able to utilize marker-assisted selection successfully in a breeding program, the molecular markers should be consistently found in various environments and show a large effect on the trait. In this study, we have considered only the first four years of a tree's life, and the phenotypic assessment was undertaken in a single environment. Therefore, further QTL analysis under different environmental conditions over the years is necessary for providing additional insights on the pattern and stability of the growth QTLs.

\section{Experimental Section}

\subsection{Plant Material}

The population consisted of $152 \mathrm{~F}_{1}$ individuals that resulted from the cross between a wild genotype Xiaoye and a cultivar Qinzhong No.1. Controlled pollination was carried out in the spring of 2009 at Yantuo, Lingbao, Henan, and seeds were collected in October and stored at $4{ }^{\circ} \mathrm{C}$. In March 2010, seeds were sown in a substrate with humus, sand and soil (1:1:1 mix) in plastic cups. Subsequently, seedlings were transplanted to the flat in a greenhouse when they had grown to a height of approximately $20 \mathrm{~cm}$. The progenies were planted in the field in March 2011 at the nursery of Northwest A\&F University, Yangling, Shaanxi. The $F_{1}$ population was designated as "DZ0901".

\subsection{DNA Extraction}

DNA was extracted from young leaves of the $152 \mathrm{~F}_{1}$ individuals and the two parental trees according to a modified CTAB procedure [44]. DNA quality was visually assessed on a $1 \%$ agarose gel by electrophoresis, and the concentration was determined using a NanoDrop ND-1000 spectrophotometer (NanoDrop Technologies Inc., Wilmington, DE, USA).

\subsection{SRAP Analysis}

SRAP analysis was performed according to $\mathrm{Li}$ and Quiros [23] with some modifications. Approximately $50 \mathrm{ng}$ DNA was added to a mixture containing $2.5 \mathrm{mM} \mathrm{MgCl} 2,0.2 \mathrm{mM}$ dNTPs, $0.4 \mathrm{mM}$ of each primer, $1 \times$ PCR buffer and $1.5 \mathrm{U}$ Taq DNA polymerase for a total volume of $25 \mu \mathrm{L}$. PCR parameters were as follows: $5 \mathrm{~min}$ at $94{ }^{\circ} \mathrm{C}, 5$ cycles of $94{ }^{\circ} \mathrm{C}$ for $1 \mathrm{~min}, 35{ }^{\circ} \mathrm{C}$ for $1 \mathrm{~min}$ and $72{ }^{\circ} \mathrm{C}$ for $1.5 \mathrm{~min}, 30$ cycles of $94{ }^{\circ} \mathrm{C}$ for $1 \mathrm{~min}, 50{ }^{\circ} \mathrm{C}$ for $1 \mathrm{~min}$ and $72{ }^{\circ} \mathrm{C}$ for $1.5 \mathrm{~min}$, and a final extension of $10 \mathrm{~min}$ at $72{ }^{\circ} \mathrm{C}$. DNA fragments were separated by electrophoresis on $8 \%$ non-denaturing 
polyacrylamide gel and visualized by silver staining. The SRAP primers used in this study are listed in Table 1.

\subsection{AFLP Analysis}

AFLP analysis consisting of genomic DNA digestion with EcoRI and MseI restriction enzymes, adapter ligation, pre-amplification, and selective amplification using EcoRI plus three and MseI plus three selective nucleotide primers were similar to those from Vos et al. [45] with modifications described by Wang et al. [46]. The following cycling parameters were used for pre-amplification: $94{ }^{\circ} \mathrm{C}$ for $2 \mathrm{~min}, 30$ cycles of $94{ }^{\circ} \mathrm{C}$ for $30 \mathrm{~s}, 56^{\circ} \mathrm{C}$ for $30 \mathrm{~s}$ and $72{ }^{\circ} \mathrm{C}$ for $80 \mathrm{~s}$, and a final extension of $5 \mathrm{~min}$ at $72{ }^{\circ} \mathrm{C}$. PCR procedure for selective amplification was as follows: $94{ }^{\circ} \mathrm{C}$ for $2 \mathrm{~min}$, 14 cycles of $94{ }^{\circ} \mathrm{C}$ for $30 \mathrm{~s}, 65^{\circ} \mathrm{C}$ for $30 \mathrm{~s}$ (reduced by $0.7{ }^{\circ} \mathrm{C} /$ cycle) and $72{ }^{\circ} \mathrm{C}$ for $80 \mathrm{~s}, 23$ cycles of $94{ }^{\circ} \mathrm{C}$ for $30 \mathrm{~s}$, $56{ }^{\circ} \mathrm{C}$ for $30 \mathrm{~s}$ and $72{ }^{\circ} \mathrm{C}$ for $80 \mathrm{~s}$, followed by $5 \mathrm{~min}$ at $72{ }^{\circ} \mathrm{C}$. DNA fragments were separated by electrophoresis on $6 \%$ denaturing polyacrylamide gel and visualized by silver staining. The AFLP primers used in this study are listed in Table 2.

\subsection{ISSR Analysis}

The protocols of Zietkiewicz et al. [47] for ISSR were adapted. Reaction mixture was as described above for SRAP except that a single primer was used. Thermal cycling conditions were as follows: $94{ }^{\circ} \mathrm{C}$ for $4 \mathrm{~min}$, followed by 38 cycles of $94{ }^{\circ} \mathrm{C}$ for $30 \mathrm{~s}, 45 \mathrm{~s}$ at the locus-specific annealing temperature and $72{ }^{\circ} \mathrm{C}$ for $1.5 \mathrm{~min}$, and then a final extension step of $72{ }^{\circ} \mathrm{C}$ for $5 \mathrm{~min}$. PCR products were detected as described above for SRAP. The 100 primers were from the \#9 ISSR primer kit (801-900) of the Biotechnology Laboratory, University of British Columbia (UBC, Vancouver, BC, Canada).

\subsection{SSR Analysis}

The SSR reaction mixture was as described above for SRAP. Thermal cycling conditions were described by Deng et al. [48]: 4 min at $94{ }^{\circ} \mathrm{C}$, locus-specific amplification cycles of $50 \mathrm{~s}$ at $94{ }^{\circ} \mathrm{C}, 50 \mathrm{~s}$ at the locus-specific annealing temperature and $90 \mathrm{~s}$ at $72{ }^{\circ} \mathrm{C}$, and a final extension step for $10 \mathrm{~min}$ at $72{ }^{\circ} \mathrm{C}$. PCR products were detected as described above for AFLP. Nineteen SSR primer combinations developed for E. ulmoides by Deng et al. [48] were used in this study.

\subsection{Segregation Analysis and Map Construction}

Data of segregating markers was analyzed as a "cross-pollinated" population using JoinMap 4.0 [49]. Deviation from expected Mendelian ratio was determined using a chi-square test. The marker placement was determined using a minimum LOD threshold of 4.0 (Plant Research International B.V. and Kyazma B.V., Wageningen, Gelderland, The Netherlands, 2006), a recombination fraction threshold of 0.45 , ripple value of 1.0 and jump threshold of 5.0, and mapping distances were calculated using the Kosambi (Plant Research International B.V. and Kyazma B.V., Wageningen, Gelderland, The Netherlands, 2006) mapping function. The genetic linkage map was plotted using MapChart 2.2 [50]. To estimate observed genome coverage, the expected genome length of each linkage group was calculated by multiplying the observed length by $(m+1) /(m-1)$, where $m$ is the number of markers in 
that linkage group, and the estimated genome length was the sum of revised length of all linkage groups [51]. Observed genome coverage was assessed by dividing the observed genome length by the estimated genome length.

\subsection{Growth Traits Assessment and QTL Analysis}

Height and basal diameter were measured to evaluate the growth of progenies in October from 2010-2013. The descriptive statistics, the skewness of the distributions and Pearson correlations of traits were calculated using SPSS 13.0 (SPSS Inc., Chicago, IL, USA, 2004) for Windows. QTL analysis was done using MapQTL 5.0 (Plant Research International B.V. and Kyazma B.V., Wageningen, Gelderland, The Netherlands, 2004) [52]. Kruskal-Wallis nonparametric test, interval mapping (IM) and multiple QTL mapping (MQM) were performed for each trait. In MQM, the markers closest to the QTL peaks detected by IM were used as cofactors. The limit of detection (LOD) thresholds was estimated with a 1000-permutation test. The QTLs with LOD values higher than the genome-wide threshold at $p<0.05$ were considered significant. However, those QTLs with a LOD score greater than 3 and smaller than the threshold were also reported. The genetic linkage map and QTL positions were drawn using MapChart 2.2 [50].

\section{Conclusions}

In this study, we report a genetic linkage map of E. ulmoides constructed by SRAP, AFLP, ISSR and SSR markers. This genetic linkage map provided an adequate coverage of the E. ulmoides genome for QTL analysis. A saturated genetic linkage map will be constructed by adding more co-dominant and functional markers. The QTL analysis provided a better genetic understanding for growth-related traits of E. ulmoides seedlings. Projects have been initiated to use the genetic linkage map to identify QTLs controlling other biological and economically important traits, and this will allow the potential of marker-assisted selection in the improvement of E. ulmoides cultivars.

\section{Acknowledgments}

This study was supported by the Special Fund for Forestry Scientific Research in the Public Interest of China (201204605).

\section{Conflicts of Interest}

The authors declare no conflict of interest.

\section{References}

1. Tippo, O. The comparative anatomy of the secondary xylem and the phylogeny of the Eucomiaceae. Am. J. Bot. 1940, 27, 832-838.

2. Kwan, C.Y.; Chen, C.X.; Deyama, T.; Nishibe, S. Endothelium-dependent vasorelaxant effects of the aqueous extracts of Eucommia ulmoides Oliv. Leaf and bark: Implications on their anti-hypertensive action. Vascul. Pharmacol. 2004, 40, 229-235. 
3. Lee, M.K.; Kim, M.J.; Cho, S.Y.; Park, S.A.; Park, K.K.; Jung, U.J.; Park, H.M.; Choi, M.S. Hypoglycemic effect of Du-zhong (Eucommia ulmoides Oliv.) leaves in streptozotocin-induced diabetic rats. Diabetes Res. Clin. Pract. 2005, 67, 22-28.

4. Hsieh, C.L.; Yen, G.C. Antioxidant actions of Du-zhong (Eucommia ulmoides Oliv.) toward oxidative damage in biomolecules. Life Sci. 2000, 66, 1387-1400.

5. Nakamura, T.; Nakazawa, Y.; Onizuka, S.; Satoh, S.; Chiba, A.; Sekihashi, K.; Miura, A.; Yasugahira, N.; Sasaki, Y.F. Antimutagenicity of Tochu tea (an aqueous extract of Eucommia ulmoides leaves): 1. The clastogen-suppressing effects of Tochu tea in CHO cells and mice. Mutat. Res. 1997, 388, 7-20.

6. Nakazawa, Y.; Bamba, T.; Takeda, T.; Uefuji, H.; Harada, Y.; Li, X.H.; Chen, R.; Inoue, S.; Tutumi, M.; Shimizu, T.; et al. Production of Eucommia-rubber from Eucommia ulmoides Oliv. (Hardy Rubber Tree). Plant Biotechnol. 2009, 26, 71-79.

7. Zhang, K.J.; Su, Y.Q.; Zhang, T. Selection and Breeding of Superior Clones of Chinese Eucommia Ulmoides; Northwest A\&F University Press: Yangling, China, 2002; pp. 9-14.

8. Tsarouhas, V.; Gullberg, U.; Lagercrantz, U. An AFLP and RFLP linkage map and quantitative trait locus (QTL) analysis of growth traits in Salix. Theor. Appl. Genet. 2002, 105, 277-288.

9. Doucleff, M.; Jin, Y.; Gao, F.; Riaz, S.; Krivanek, A.F.; Walker, M.A. A genetic linkage map of grape, utilizing Vitis rupestris and Vitis arizonica. Theor. Appl. Genet. 2004, 109, 1178-1187.

10. Venkateswarlu, M.; Urs, S.R.; Nath, B.S.; Shashidhar, H.E.; Maheswaran, M.; Veeraiah, T.M.; Sabitha, M.G. A first genetic linkage map of mulberry (Morus spp.) using RAPD, ISSR, and SSR markers and pseudotestcross mapping strategy. Tree Genet. Genomes 2006, 3, 15-24.

11. Pakull, B.; Groppe, K.; Meyer, M.; Markussen, T.; Fladung, M. Genetic linkage mapping in aspen (Populus tremula L. and Populus tremuloides Michx.). Tree Genet. Genomes 2009, 5, 505-515.

12. Gulsen, O.; Uzun, A.; Canan, I.; Seday, U.; Canihos, E. A new citrus linkage map based on SRAP, SSR, ISSR, POGP, RGA and RAPD markers. Euphytica 2010, 173, 265-277.

13. Wang, Y.; Sun, X.; Tan, B.; Zhang, B.; Xu, L.A.; Huang, M.; Wang, M. A genetic linkage map of Populus adenopoda Maxim. $\times$ P. alba L. hybrid based on SSR and SRAP markers. Euphytica 2010, 173, 193-205.

14. Fernandez-Fernandez, F.; Antanaviciute, L.; van Dyk, M.M.; Tobutt, K.R.; Evans, K.M.; Rees, D.J.G.; Dunwell, J.M.; Sargent, D.J. A genetic linkage map of an apple rootstock progeny anchored to the Malus genome sequence. Tree Genet. Genomes 2012, 8, 991-1002.

15. Souza, L.M.; Gazaffi, R.; Mantello, C.C.; Silva, C.C.; Garcia, D.; le Guen, V.; Cardoso, S.E.A.; Garcia, A.A.F.; Souza, A.P. QTL mapping of growth-related traits in a full-Sib family of rubber tree (Hevea brasiliensis) evaluated in a sub-tropical climate. PLoS One 2013, 8, e61238.

16. Garcia, M.R.; Asins, M.J.; Carbonell, E.A. QTL analysis of yield and seed number in Citrus. Theor. Appl. Genet. 2000, 101, 487-493.

17. Scalfi, M.; Troggio, M.; Piovani, P.; Leonardi, S.; Magnaschi, G.; Vendramin, G.G.; Menozzi, P. A RAPD, AFLP and SSR linkage map, and QTL analysis in European beech (Fagus sylvatica L.). Theor. Appl. Genet. 2004, 108, 433-441.

18. Freeman, J.S.; Whittock, S.P.; Potts, B.M.; Vaillancourt, R.E. QTL influencing growth and wood properties in Eucalyptus globulus. Tree Genet. Genomes 2009, 5, 713-722. 
19. Thumma, B.R.; Baltunis, B.S.; Bell, J.C.; Emebiri, L.C.; Moran, G.F.; Southerton, S.G. Quantitative trait locus (QTL) analysis of growth and vegetative propagation traits in Eucalyptus nitens full-sib families. Tree Genet. Genomes 2010, 6, 877-889.

20. Samils, B.; Ronnberg-Wastljung, A.C.; Stenlid, J. QTL mapping of resistance to leaf rust in Salix. Tree Genet. Genomes 2011, 7, 1219-1235.

21. Sadok, I.B.; Celton, J.M.; Essalouh, L.; El Aabidine, A.Z.; Garcia, G.; Martinez, S.; Grati-Kamoun, N.; Rebai, A.; Costes, E.; Khadari, B. QTL mapping of flowering and fruiting traits in olive. PLoS One 2013, 8, e62831.

22. Dong, J.; Ma, X.H.; Wei, Q.; Peng, S.B.; Zhang, S.C. Effects of growing location on the contents of secondary metabolites in the leaves of four selected superior clones of Eucommia ulmoides. Ind. Crop. Prod. 2011, 34, 1607-1614.

23. Li, G.; Quiros, C.F. Sequence-related amplified polymorphism (SRAP), a new marker system based on a simple PCR reaction: Its application to mapping and gene tagging in Brassica. Theor. Appl. Genet. 2001, 103, 455-461.

24. Grando, M.S.; Bellin, D.; Edwards, K.J.; Pozzi, C.; Stefanini, M.; Velasco, R. Molecular linkage maps of Vitis vinifera L. and Vitis riparia Mchx. Theor. Appl. Genet. 2003, 106, 1213-1224.

25. Zhang, R.; Wu, J.; Li, X.G.; Khan, M.A.; Chen, H.; Korban, S.S.; Zhang, S.L. An AFLP, SRAP, and SSR genetic linkage map and identification of QTLs for fruit traits in pear (Pyrus L.). Plant Mol. Biol. Report. 2013, 31, 678-687.

26. Sanchez-Robles, J.M.; Balao, F.; Garcia-Castano, J.L.; Terrab, A.; Navarro-Sampedro, L.; Talavera, S. Nuclear microsatellite primers for the endangered relict fir, Abies pinsapo (pinaceae) and cross-amplification in related mediterranean species. Int. J. Mol. Sci. 2012, 13, 14243-14250.

27. Wang, H.; Chen, N.F.; Zheng, J.Y.; Wang, W.C.; Pei, Y.Y.; Zhu, G.P. Isolation and characterization of eleven polymorphic microsatellite loci for the valuable medicinal plant Dendrobium huoshanense and cross-species amplification. Int. J. Mol. Sci. 2012, 13, 16779-16784.

28. Kang, B.Y.; Major, J.E.; Rajora, O.P. A high-density genetic linkage map of a black spruce (Picea mariana) $\times$ red spruce (Picea rubens) interspecific hybrid. Genome 2011, 54, 128-143.

29. Lorieux, M.; Goffinet, B.; Perrier, X.; Gonzalez de Leon D.; Lanaud, C. Maximum-likelihood models for mapping genetic markers showing segregation distortion. 1. Backcross populations. Theor. Appl. Genet. 1995, 90, 73-80.

30. Sauge, M.H.; Lambert, P.; Pascal, T. Co-localisation of host plant resistance QTLs affecting the performance and feeding behaviour of the aphid Myzus persicae in the peach tree. Heredity 2012, 108, 292-301.

31. Jenczewski, E.; Gherardi, M.; Bonnin, I.; Prosperi, J.M.; Olivieri, I.; Huguet, T. Insight on segregation distortions in two intraspecific crosses between annual species of Medicago (Leguminosae). Theor. Appl. Genet. 1997, 94, 682-691.

32. Kasha, K.J.; Kao, K.N. High frequency haploid production in barley (Hordeum vulgare). Nature 1970, 225, 874-876.

33. Bradshaw, H.D.; Stettler, R.F. Molecular genetics of growth and development in Populus. II. Segregation distortion due to genetic load. Theor. Appl. Genet. 1994, 89, 551-558. 
34. Fishman, L.; Willis, J.H. A novel meiotic drive locus almost completely distorts segregation in Mimulus (monkeyflower) hybrids. Genetics 2005, 169, 347-353.

35. Fishman, L.; Kelly, A.J.; Morgan, E.; Willis, J.H. A genetic map in the Mimulus guttatus species complex reveals transmission ratio distortion due to heterospecific interactions. Genetics 2001, $159,1701-1716$.

36. Fishman, L.; Aagaard, J.; Tuthill, J.C. Toward the evolutionary genomics of gametophytic divergence: Patterns of transmission ratio distortion in monkey flower (Mimulus) hybrids reveal a complex genetic basis for conspecific pollen precedence. Evolution 2008, 62, 2958-2970.

37. Bradshaw, H.D.; Stettler, R.F. Molecular genetics of growth and development in Popolus. IV. Mapping QTLs with large effects on growth, form and phenology traits in a forest tree. Genetics 1995, 139, 963-973.

38. Zhang, D.; Zhang, Z.; Yang, K. QTL analysis of growth and wood chemical content traits in an interspecific backcross family of white poplar (Populus tomentosa $\times$ P. bolleana $) \times$ P. tomentosa . Can. J. For. Res. 2006, 36, 2015-2023.

39. Ronnberg-Wastljung, A.C.; Glynn, C.; Weih, M. QTL analyses of drought tolerance and growth for a Salix dasyclados $\times$ Salix viminalis hybrid in contrasting water regimes. Theor. Appl. Genet. 2005, 110, 537-549.

40. Verhaegen, D.; Plomion, C.; Gion, J.M.; Poitel, M.; Costa, P.; Kremer, A. Quantitative trait dissection analysis in Eucalyptus using RAPD markers. 1. Detection of QTL in interspecific hybrid progeny, stability of QTL expression across different ages. Theor. Appl. Genet. 1997, 95, 597-608.

41. Kenis, K.; Keulemans, J. Study of tree architecture of apple (Malus $\times$ domestica Borkh.) by QTL analysis of growth traits. Mol. Breed. 2007, 19, 193-208.

42. Emebiri, L.C.; Devey, M.E.; Matheson, A.C.; Slee, M.U. Age-related changes in the expression of QTLs for growth in radiata pine seedlings. Theor. Appl. Genet. 1998, 97, 1053-1061.

43. Stoeckli, S.; Mody, K.; Gessler, C.; Patocchi, A.; Jermini, M.; Dorn, S. QTL analysis for aphid resistance and growth traits in apple. Tree Genet. Genomes 2008, 4, 833-847.

44. Porebski, S.; Bailey, L.G.; Baum, B.R. Modification of a CTAB DNA extraction protocol for plants containing high polysaccharide and polyphenol components. Plant Mol. Biol. Rep. 1997, $15,8-15$.

45. Vos, P.; Hoger, R.; Blecker, M. AFLP: A new technique for DNA fingerprinting. Nucleic Acids Res. 1995, 23, 4407-4414.

46. Wang, D.W.; Li, Y.; Li, Z.Q. Identification of a male-specific amplified fragment length polymorphism (AFLP) and a sequence characterized amplified region (SCAR) Marker in Eucommia ulmoides Oliv. Int. J. Mol. Sci. 2011, 12, 857-864.

47. Zietkiewicz, E.; Rafalski, A.; Labuda, D. Genome fingerprinting by simple sequence repeats (SSR)-anchored PCR amplification. Genomics 1994, 20, 176-183.

48. Deng, J.Y.; Liu, Y.F.; Huang, H.W. Development and characterization of microsatellite markers in Eucommia ulmoides Oliver (Eucommiaceae). Mol. Ecol. Notes 2006, 6, 496-498.

49. Van Ooijen, J.W. Joinmap 4, Software for the Calculation of Genetic Linkage Maps in Experimental Populations; Plant Research International B.V. and Kyazma B.V.: Wageningen, The Netherlands, 2006. 
50. Voorrips, R.E. MapChart: Software for the graphical presentation of linkage maps and QTLs. J. Hered. 2002, 93, 77-78.

51. Chakravarti, A.; Lasher, L.K.; Reefer, J.E. A maximum-likelihood method for estimating genome length using genetic linkage data. Genetics 1991, 128, 175-182.

52. Van Ooijen, J.W. Mapqtl 5, Software for the Mapping of Quantitative Trait Loci in Experimental Populations; Plant Research International B.V. and Kyazma B.V.: Wageningen, The Netherlands, 2004.

(C) 2014 by the authors; licensee MDPI, Basel, Switzerland. This article is an open access article distributed under the terms and conditions of the Creative Commons Attribution license (http://creativecommons.org/licenses/by/3.0/). 